\title{
Determination of porphyrins in oral bacteria by liquid chromatography electrospray ionization tandem mass spectrometry
}

\author{
Jonas Fyrestam ${ }^{1}$ - Nadja Bjurshammar ${ }^{2}$ - Elin Paulsson ${ }^{3}$ - Annsofi Johannsen ${ }^{2}$. \\ Conny Östman ${ }^{1}$
}

Received: 22 April 2015 / Revised: 12 June 2015 / Accepted: 16 June 2015 / Published online: 14 July 2015

(C) The Author(s) 2015. This article is published with open access at Springerlink.com

\begin{abstract}
Biofilms in the oral cavity can be visualized by fluorescence and a common assumption is that the endogenously produced porphyrins in certain bacteria give rise to this fluorescence. Porphyrin content in oral bacteria has been sparingly investigated, and non-selective detection techniques such as utilizing the Soret fluorescence band of porphyrins are often used. In the present study, a quantitative and selective method for the determination of porphyrins in oral bacteria has been developed and validated using high performance liquid chromatography-tandem mass spectrometry. Lysis of bacteria using Tris-EDTA buffer together with ultrasonication showed high microbial killing efficiency $\geq 99.98 \%$, and sample clean-up using $\mathrm{C}_{18^{-}}$ solid phase extraction resulted in low matrix effects $\leq 14 \%$ for all analytes. Using this method, the porphyrin content was determined in the two oral pathogens Aggregatibacter actinomycetemcomitans and Porphyromonas gingivalis, as well as for baker's yeast, Saccharomyces cerevisiae. Uroporphyrin, 7-carboxylporphyrin, 6-carboxylporphyrin,
\end{abstract}

Electronic supplementary material The online version of this article (doi:10.1007/s00216-015-8864-2) contains supplementary material, which is available to authorized users.

Conny Östman

conny.ostman@aces.su.se

1 Department of Environmental Science and Analytical Chemistry, Analytical and Toxicological Chemistry, 10691 Stockholm, Sweden

2 Department of Dental Medicine, Karolinska Institutet, 14104 Huddinge, Sweden

3 Department of Aquatic Sciences and Assessment, Swedish University of Agricultural Sciences, 75007 Uppsala, Sweden coproporphyrin, and protoporphyrin IX were identified in the investigated microorganisms, and it was shown that the porphyrin profile differs between the two bacteria, as well as for $S$. cerevisiae. To our knowledge, this is the first time the porphyrin profile has been determined for the bacterium A. actinomycetemcomitans.

Keywords HPLC/MS/MS · Aggregatibacter actinomycetemcomitans $\cdot$ Porphyromonas gingivalis . Saccharomyces cerevisiae · Porphyrins · Oral bacteria

$\begin{array}{ll}\text { Abbreviations } \\ \text { 5PI } & \text { Pentacarboxylic porphyrin I } \\ \text { 6PI } & \text { Hexacarboxylic porphyrin I } \\ \text { 7PI } & \text { Heptacarboxylporphyrin I } \\ \text { CFU } & \text { Colony forming units } \\ \text { CPI } & \text { Coproporphyrin I } \\ \text { CPIII } & \text { Coproporphyrin III } \\ \text { MPIX } & \text { Mesoporphyrin IX } \\ \text { PPIX } & \text { Protoporphyrin IX } \\ \text { S/N } & \text { Signal to noise } \\ \text { SRM } & \text { Selected reaction monitoring } \\ \text { TE } & \text { Tris/EDTA } \\ \text { UPI } & \text { Uroporphyrin I }\end{array}$

Introduction

Porphyrins are a group of macromolecules involved in the biosynthesis of several essential biological molecules such as heme and chlorophyll $[1,2]$. The basic structure for all porphyrins is porphine, a tetrapyrrolic ring linked by methine bridges. The individual porphyrins are specified by the side chain substituents including alkyl, alkene, or carboxylic acid 
groups. Owing to their aromatic nature, porphyrins act as pigments that are fluorescent with characteristic bands of absorbance between $\lambda=390-425 \mathrm{~nm}$ [3], a strong Soret band in the $\lambda=380-500 \mathrm{~nm}$ region, and weaker Q-bands in the $\lambda=500$ $750 \mathrm{~nm}$ region.

Porphyrins have been determined in a variety of matrices, including blood, urine, feces, and also in bacteria [4-7]. The commonly used method for separation of porphyrins is reversed phase high-performance liquid chromatography (RPHPLC) using methanol or acetonitrile as the organic phase modified with a buffer such as ammonium acetate-acetic acid $[2,5,8-10]$. The fluorescent nature of the porphyrins is most frequently used for detection $[6,8,11]$, but mass spectrometry (MS) has also been used in a number of studies [3-5]. Determination of porphyrins in bacteria has mostly been performed using fluorescence detection, utilizing the similarities of the fluorescence spectral properties of porphyrins [7, 12, 13]. This means that detection of porphyrins with fluorescence is a nonselective method. However, when determining porphyrins in biological samples, the number of porphyrin species and the complex nature of the matrix demand a selective detection technique. This can be achieved by using tandem mass spectrometry.

Detection of oral biofilm and early signs of caries can be made with fluorescence techniques, and dental biofilm can be visualized as red fluorescence when the teeth are illuminated with light in the blue spectral region [14-16]. Studies have suggested that obligate anaerobic bacteria are responsible for this red fluorescence because of their increasing numbers in the mature oral biofilm $[16,17]$. In a previous study, our research group has shown that also isolated colonies of Aggregatibacter actinomycetemcomitans are capable of emiting red fluorescence when exposed to blue light $(\lambda=$ $370 \mathrm{~nm}$ ) and suggested that this fluorescence emission is due to endogenously produced porphyrins [18]. The porphyrin content of the bacterium Propionibacterium acnes has been investigated in several studies [12, 13, 19, 20], but little work has been done on oral pathogens. Soukos et al. [7] have analyzed the porphyrin content of oral black-pigmented bacteria Porphyromonas gingivalis, Prevotella intermedia, Prevotella nigrescens, and Prevotella melaninogenica using RP-HPLC with fluorescence detection.

Studies have demonstrated that bacteria containing porphyrins are sensitive to visible light, in the blue as well as the red spectral region [21, 22]. It has been proposed that oral bacteria can be eradicated using light in the blue region of the visible spectrum, involving a mechanism that is supposed to be based on optical excitation of the bacteria's endogenous porphyrins [23]. This is used in phototherapy methods (i.e., treatment by visible light without any added exogenous photosensitizer). Since bacterial resistance to antibiotics is increasing, there is a growing interest for alternative treatments of microbial infections. For this reason, it would be of great interest to investigate the content of porphyrins in oral pathogens associated with periodontal disease as well as in oral biofilm, in order to connect bacterial susceptibility to phototoxicity with their content of porphyrins.

The aim of this study was to develop a method for the sensitive and selective determination of porphyrins in oral pathogenic bacteria by combining an efficient extraction and clean-up method for oral bacteria with efficient separation on RP-HPLC and selective detection with mass spectrometry using selected reaction monitoring (SRM). A. actinomycetemcomitans and $P$. gingivalis were selected as model oral pathogens since they are periodontopathogenic, non-fastidious anaerobic bacteria that are comparably easy to culture. Saccharomyces cerevisiae was used as an additional model organism for validation of the method.

\section{Material and methods}

\section{Chemicals and reagents}

HPLC grade methanol and acetonitrile were purchased from Rathburn Chemicals Ltd. (Walkerburn, Scotland). Formic acid ( $\geq 98 \%$ ), acetic acid ( $\geq 99.8 \%$ ), and hydrochloric acid ( $\geq 37 \%$ ) were obtained from Sigma Aldrich (Schnelldorf, Germany). Deionized water at $18 \Omega$ was produced by a Synergy 185 water purification system from Millipore (Molsheim, France). Ammonium acetate, sodium bicarbonate, and tris(hydroxymethyl)aminomethane (Tris) were all of pro analysi grade and obtained from Merck (Darmstadt, Germany). Dimethylformamide (DMF) was obtained from VWR International (Fount-Sous-Bois, France) and ethylenediaminetetraacetic acid (EDTA) disodium dihydrate salt of reagent grade from Scherlab S.L. (Sentmenat, Spain). $S$. cerevisiae (commonly known as Baker's yeast) was used for method validation. It was obtained from Jästbolaget (Sollentuna, Sweden).

Porphyrin standards were purchased as individual compounds and as a chromatographic marker kit (Table 1) from Frontier Scientific, Logan, UT, USA (part No. CMK-1A). The structure of protoporphyrin IX is shown in Fig. 1.

\section{Preparation of solutions and standards}

A $1 \mathrm{M}$ ammonium acetate buffer was prepared by dissolving ammonium acetate in deionized water and adjusting $\mathrm{pH}$ to 5.16 by addition of acetic acid. TE-buffer was made by mixing a $1 \mathrm{M}$ solution of Tris with a $0.1 \mathrm{M}$ EDTA $(\mathrm{pH} 8)$ solution to give concentrations of $5 \mathrm{mM}$ Tris and $10 \mathrm{mM}$ EDTA adjusting the $\mathrm{pH}$ to 7.2 using $\mathrm{HCl}$.

The chromatographic marker kit was dissolved in $6 \mathrm{M}$ formic acid to obtain a stock solution with a concentration of $2 \mu \mathrm{mol} / \mathrm{L}$ for each of uroporphyrin I (UPI), 7 - 
Table 1 Abbreviations, CAS no, purities, molecular weights, and manufacturers for the investigated porphyrins

\begin{tabular}{llllll}
\hline Compound & Abbreviation & CAS no. & Purity (\%) & Mw (Da) & Manufacturer \\
\hline Uroporphyrin I & UP I & $607-14-7$ & $\geq 90$ & 830.75 & Frontier Scientific Inc. \\
7-Carboxylporphyrin I & 7P I & $65406-45-3$ & $\geq 90$ & 786.74 & Frontier Scientific Inc. \\
6-Carboxylporphyrin I & 6P I & $73913-56-1$ & $\geq 90$ & 742.73 & Frontier Scientific Inc. \\
5-Carboxylporphyrin I & 5P I & $28100-66-5$ & $\geq 90$ & 698.72 & Frontier Scientific Inc. \\
Coproporphyrin I & CP I & $531-14-6$ & $\geq 90$ & 654.71 & Frontier Scientific Inc. \\
Coproporphyrin III & CP III & $14643-66-4$ & $\geq 97$ & 654.71 & Frontier Scientific Inc. \\
Mesoporphyrin IX & MP IX & $493-90-3$ & $\geq 90$ & 566.69 & Frontier Scientific Inc. \\
Mesoporphyrin IX dihydrochloride & MP IX Cl & $68938-72-7$ & 95 & 639.61 & Sigma Aldrich \\
Protoporphyrin IX & PP IX & $553-12-8$ & $\geq 95$ & 562.66 & Sigma Aldrich \\
Hemin & Hemin & $16009-13-5$ & $\geq 90$ & 651.94 & Sigma Aldrich \\
\hline
\end{tabular}

carboxylporphyrin I (7PI), 6-carboxylporphyrin I (6PI), 5carboxylporphyrin I (5PI), coproporphyrin I (CPI), and mesoporphyrin IX (MPIX). Separate stock solutions with concentrations between 0.2 and $0.3 \mathrm{mmol} / \mathrm{L}$ were prepared of MPIX, protoporphyrin IX (PPIX), and coproporphyrin III (CPIII). This was made in DMF:methanol 1:1 $(v / v)$ because of the higher hydrophobicity of these compounds. Working standards were prepared in $6 \mathrm{M}$ formic acid from the stock solutions. Stability tests in $6 \mathrm{M}$ formic acid showed that the more hydrophobic compounds PPIX and MPIX were unstable in a polar solvent (data not shown), which made daily preparation of working standards necessary. This instability is

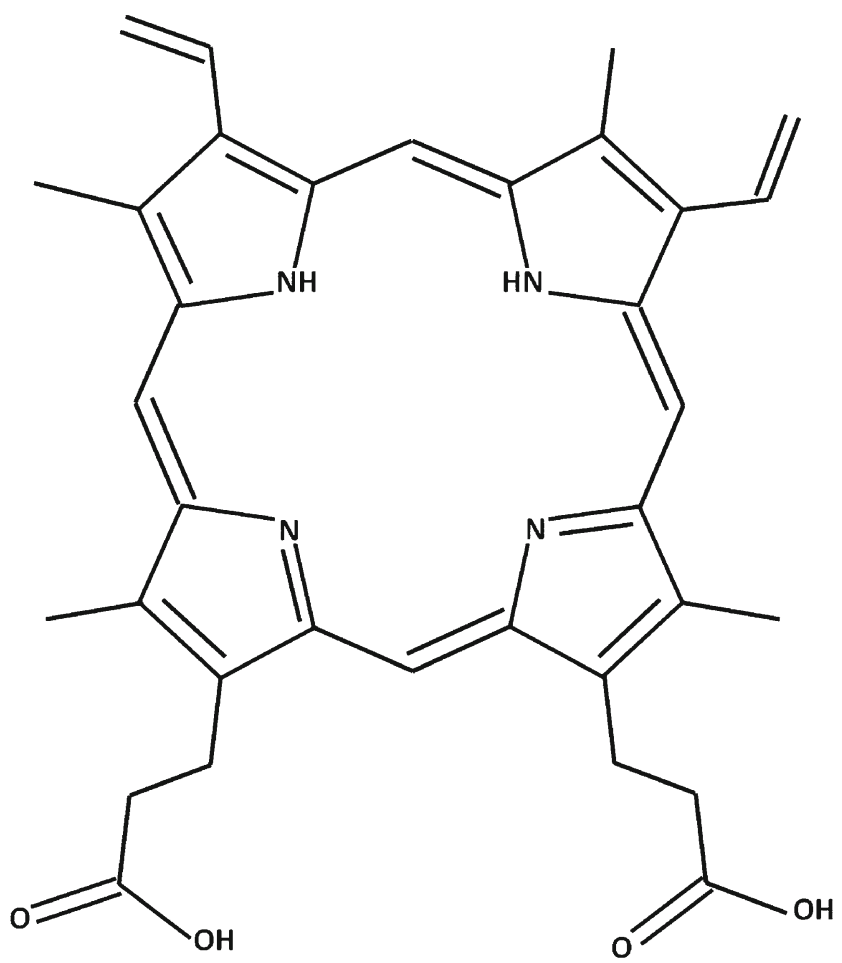

Fig. 1 Structure of protoporphyrin IX (3,7,12,17-tetramethyl-8,13divinyl-2,18-porphinedipropionic acid) probably due to the aggregation of the porphyrins in aqueous solution [24].

\section{Culturing and harvesting bacteria}

A. actinomycetemcomitans strain ATCC 33384 serotype c and $P$. gingivalis strain ATCC BAA-308/W83, both obtained from the Department of Clinical Bacteriology at the University of Gothenburg, were cultivated in a $\mathrm{CO}_{2}$ incubator, model T303 (Assab Medicine, Stockholm, Sweden). A. actinomycetemcomitans was grown in a $4.9 \% \mathrm{CO}_{2}$ atmosphere and $P$. gingivalis was grown under anaerobic conditions, both at a temperature of $37.1{ }^{\circ} \mathrm{C}$. A. actinomycetemcomitans was grown on Columbia agar with $5 \%$ defibrinated horse blood and $P$. gingivalis on Brucella agar containing horse blood, hemin, and K-vitamin (Substrate unit, Karolinska University Hospital, Huddinge). Subculturing was made by incubation for $4 \mathrm{~d}$ on blood agar plates followed by transfer to new blood agar plates and grown for an additional $7 \mathrm{~d}$ for A. actinomycetemcomitans and $12 \mathrm{~d}$ for $P$. gingivalis. The bacteria were harvested from the agar plates using a sterile plastic cell scraper and transferred to $500 \mu \mathrm{L}$ of $0.4 \% \mathrm{NaCl}$ in a $1.5 \mathrm{~mL}$ Eppendorf vial and the sample size measured gravimetrically.

\section{Sample preparation}

The harvested bacterial cell material was transferred to a test tube, vortexed for $30 \mathrm{~s}$, and pelleted by centrifugation for $10 \mathrm{~min}$ at $2500 \mathrm{~g}$. The supernatant was removed, $1000 \mu \mathrm{L}$ of TE-buffer was added, and the pellet was resuspended by vortexing. The test tube was protected from light by wrapping in aluminum foil and put to digest for $1 \mathrm{~h}$ at room temperature, after which proteins were precipitated by addition of $1000 \mu \mathrm{L}$ of $6 \mathrm{M}$ formic acid. The cells were then further lysed using an ultrasonication rod for $1 \mathrm{~min}$. The sample was subsequently centrifuged for $10 \mathrm{~min}$ at $2500 \mathrm{~g}$ and the supernatant 
containing porphyrins was cleaned up using an end-capped Isolute C18 SPE- cartridge ( $3 \mathrm{~mL}, 200 \mathrm{mg}$; International Sorbent Technology Ltd., Hengoed, UK). The SPE cartridge was preconditioned with $3 \mathrm{~mL}$ of methanol:acetonitrile 9:1 $(v / v)$, $3 \mathrm{~mL}$ of deionized water, and $3 \mathrm{~mL}$ of ammonium acetate (1 M, pH 5.16). The sample was added and washed with $3 \mathrm{~mL}$ of ammonium acetate (1 M, pH 5.16). Porphyrins were eluted using $2 \mathrm{~mL}$ of acetone:formic acid, 9:1 ( $\mathrm{v} / \mathrm{v})$. The eluent was placed in a heated water bath at $70{ }^{\circ} \mathrm{C}$ and slowly evaporated to dryness under a gentle stream of nitrogen. Finally the volume was adjusted to $500 \mu \mathrm{L}$ using $6 \mathrm{M}$ formic acid.

\section{Instrumentation}

Two HPLC systems were used for analysis of the porphyrins. The first system consisted of an HPLC system from Perkin Elmer (Norwalk, CT, USA) with a binary solvent delivery system consisting of two 200 series micro pumps, a 200 series autosampler equipped with a $5 \mu \mathrm{L}$ injection loop, and a 200 series vacuum degasser, and were used for the analysis of A. actinomycetemcomitans. The second system was an Agilent HPLC system (Wilmington, DE, USA) consisting of an 1100 binary pump solvent delivery system, an 1100 degasser, and an 1100 autosampler. The latter system was used for analysis of $P$. gingivalis and $S$. cerevisiae using $5 \mu \mathrm{L}$ as injection volume. Both HPLC systems were coupled to a SCIEX API 2000 triple quadrupole mass spectrometer (Toronto, ON, Canada) equipped with a Turbolon Spray interface run in electrospray ionization mode. Analyst 1.4.2 software from SCIEX was used for instrument control and data processing.

Separation was performed on an ACE 3 C18-PFP column $\left(75 \times 2.1 \mathrm{~mm}, \mathrm{~d}_{\mathrm{p}}=3 \mu \mathrm{m}\right.$, Advanced Chromatography Technologies Ltd., Aberdeen, Scotland) with a C18-PFP guard column $(2.1 \mathrm{~mm}$ i.d.) and a mobile phase flow rate of $100 \mu \mathrm{L} /$ min. Mobile phase A consisted of $95 \%$ water, $5 \%$ acetonitrile, and $0.1 \%$ formic acid $(v / v / v)$, and mobile phase B $95 \%$ acetonitrile, $5 \%$ water, and $0.1 \%$ formic acid $(v / v / v)$. A linear gradient program was applied, increasing the mobile phase B from 30 to $50 \%$ during the first $10.0 \mathrm{~min}$, followed by a linear increase of mobile phase B up to $100 \%$ from 10.0 to $10.2 \mathrm{~min}$. The mobile phase was held at $100 \%$ B for another $24.8 \mathrm{~min}$. Before each analysis, the column was equilibrated for 20.0 min with $30 \%$ mobile phase B.

Detection of porphyrins was made in positive electrospray ionization mode $(\mathrm{ESI}+)$ and SRM with three compound-specific product ions for each porphyrin. Porphyrins were identified by retention time and the presence of all three product ions. All transitions were used for quantification. The instrument was tuned by direct infusion of standard solution with a concentration of $2 \mathrm{nmol} / \mathrm{mL}$ of each of the porphyrins in $6 \mathrm{M}$ formic acid solution. MS parameters were as follows: ion source temperature $200^{\circ} \mathrm{C}$, capillary voltage $5 \mathrm{kV}$, nebulizer gas $\left(\mathrm{N}_{2}\right) 35 \mathrm{psi}$, collision gas $\left(\mathrm{N}_{2}\right) 8 \mathrm{psi}$, and curtain gas $\left(\mathrm{N}_{2}\right) 20$ psi. The MS/MS parameters used for analysis can be seen in Table 2.

\section{Determination of microbial killing efficiency}

A. actinomycetemcomitans was grown and subcultured under the conditions described above. The bacteria were harvested at $\mathrm{d} 4$ of incubation, transferred to a test tube containing $1 \mathrm{~mL}$ of $0.4 \% \mathrm{NaCl}$, and vortexed for $30 \mathrm{~s}$ giving a stock solution of bacteria. One hundred microliters of the stock solution were added to $900 \mu \mathrm{L}$ of $0.4 \% \mathrm{NaCl}$ and vortexed, resulting in a dilution factor of $10^{-1}$. This dilution process was successively repeated, giving seven control samples with dilution factors ranging from $10^{-1}$ to $10^{-7}$. From each dilution step, $100 \mu \mathrm{L}$ were evenly distributed with a sterile glass spreader on an agar plate. The remaining $900 \mu \mathrm{L}$ of the stock solution were put in TE-buffer and treated with formic acid and ultrasonication as described above. Same dilution procedure as described above was performed and $100 \mu \mathrm{L}$ were put on agar plates. All agar plates were incubated for $4 \mathrm{~d}$ and plates containing between 30 and 300 colony-forming units (CFU) were counted to estimate the killing efficiency. The experiment was performed in triplicate for statistical evaluation.

\section{Linearity, LOD, and LOQ}

Calibration standards containing UPI, 7PI, 6PI, 5PI, CPI, MPIX, and PPIX were prepared at six concentration levels: $50,100,300,500,700$, and $900 \mathrm{pmol} / \mathrm{mL}$ in $6 \mathrm{M}$ formic acid. All calibration standards were injected in triplicate. The resulting peak area $(y)$ versus concentration $(x)$ was plotted for each porphyrin and treated by least squares method of linear regression.

For determination of LOD and LOQ, the signal to noise ratio $(\mathrm{S} / \mathrm{N})$ was calculated from injections of $25 \mathrm{fmol}$ of each porphyrin in standard solution. The noise was defined as the standard deviation of the peak area and the signal was defined as the mean area of triplicate injection. LOD and LOQ were defined as three and 10 times the $\mathrm{S} / \mathrm{N}$ ratio, respectively.

\section{Accuracy, precision, and recovery}

Accuracy was determined by analyzing triplicate samples of spiked extracts from $S$. cerevisiae with a known concentration of each porphyrin. It was calculated by the relative difference between the mean of the measured concentration and the true concentration. Endogenously produced amounts of porphyrins in the extracts were subtracted from the spiked samples. MPIX was used as a volumetric internal standard $\left(\mathrm{IS}_{\mathrm{vol}}\right)$ to correct for differences in injection volume and/or ionization efficiency between analytical runs. Spiked extracts from S. cerevisiae were analyzed in triplicate during the same day 
Table 2 Conditions for the MS/MS SRM analyses

\begin{tabular}{|c|c|c|c|c|c|c|c|c|c|c|c|}
\hline \multirow[t]{2}{*}{ Porphyrin } & \multirow{2}{*}{$\begin{array}{l}{[\mathrm{M}+\mathrm{H}]^{+}} \\
m / z\end{array}$} & \multirow{2}{*}{$\begin{array}{l}\text { Time window } \\
\text { (min) }\end{array}$} & \multicolumn{3}{|c|}{ Transition 1} & \multicolumn{3}{|c|}{ Transition 2} & \multicolumn{3}{|c|}{ Transition 3} \\
\hline & & & $m / z$ & $\mathrm{CE}(\mathrm{V})^{\mathrm{a}}$ & $\operatorname{CXP}(\mathrm{V})^{\mathrm{b}}$ & $m / z$ & $\mathrm{CE}(\mathrm{V})^{\mathrm{a}}$ & $\operatorname{CXP}(V)^{b}$ & $\mathrm{~m} / \mathrm{z}$ & $\mathrm{CE}(\mathrm{V})^{\mathrm{a}}$ & $\mathrm{CXP}(\mathrm{V})^{\mathrm{b}}$ \\
\hline Uroporphyrin I & 831 & $0.0-5.5$ & 727 & 70 & 22 & 623 & 85 & 22 & 655 & 85 & 22 \\
\hline 7-Carboxylporphyrin I & 787 & $5.5-8.2$ & 683 & 85 & 22 & 670 & 85 & 22 & 623 & 85 & 22 \\
\hline 6-Carboxylporphyrin I & 743 & $8.2-11.0$ & 639 & 70 & 22 & 507 & 90 & 22 & 521 & 90 & 22 \\
\hline 5-Carboxylporphyrin I & 699 & $11.0-13.2$ & 463 & 95 & 20 & 595 & 119 & 22 & 640 & 47 & 24 \\
\hline Coproporphyrin I & 655 & $13.2-19.5$ & 537 & 65 & 24 & 596 & 51 & 26 & 523 & 63 & 26 \\
\hline Mesoporphyrin IX & 567 & $19.5-27.5$ & 449 & 61 & 24 & 479 & 85 & 22 & 508 & 43 & 24 \\
\hline Protoporphyrin IX & 563 & $27.5-35.0$ & 445 & 65 & 22 & 504 & 45 & 22 & 489 & 89 & 22 \\
\hline
\end{tabular}

a Collision energy

${ }^{\mathrm{b}}$ Collision exit potential

to investigate the intra-day precision of the analysis and is presented as coefficient of variation $(\mathrm{CV} \%)$.

For estimation of recovery, a standard solution containing $0.5 \mathrm{nmol} / \mathrm{mL}$ of each porphyrin in $6 \mathrm{M}$ formic acid was put through the sample preparation steps described above. Detector responses from the standard mixture going through the clean-up steps were compared with the detector responses from the untreated standard solution. The experiment was performed in triplicate.

\section{Results and discussion}

\section{HPLC separation of porphyrins}

In Fig. 2, a chromatogram is shown that demonstrates the separation of a standard solution containing the seven porphyrins. They are all well separated with a resolution factor (R) higher than 1.5 (i.e., baseline separation) with a total run time of $30 \mathrm{~min}$. The elution order is UPI, 7PI, 6PI, 5PI, CPI, MPIX, and PPIX, corresponding to the decreasing number of carboxylic acid groups attached to the basic porphine structure. All porphyrins have a number of possible isomers of which type I and III are the ones commonly present. Type III isomers are formed by enzymatic action, whereas type I isomers are formed by spontaneous chemical ring closure in the absence of enzymes [3]. The separations of the type I isomers (UPI, 7PI, 6PI, and 5PI), type IX isomers of MPIX and PPIX, and both type I and type III of CP were evaluated. CP type III isomer elutes after type I and the isomers are well separated with $\mathrm{R}>1.5$. CPIII has not been validated with this method but has been assumed to have identical properties to CPI.

\section{Detection of porphyrins using ESI MS/MS}

There are large differences in the ESI+ ionization efficiency between the porphyrins. UPI contains eight carboxylic acid substituents, whereas PPIX contains only two. This could be a possible reason for the latter compound having a 40 times higher response than UPI in the Agilent/Sciex API 2000 LC/MS system. That the less polar PPIX has a higher response in ESI-MS compared with the more polar porphyrin UPI is most probably due to the various distributions of ions of different hydrophobicity in the droplets of the spray [25]. In the Perkin Elmer/Sciex API 2000 LC/MS system, the difference in response between the porphyrins was substantially lower. The retention times were different in the two systems, which meant that PPIX was ionized in different mobile-phase compositions in the two LC/ MS systems (Figs. 5 and 6). Selection of mobile phases has shown to be important when ESI is used. Ionization efficiency is influenced by the solvent composition where high water content of the mobile phase tends to give decreased ionization efficiency. This is due to the high viscosity of water, leading to difficulties in forming a stable spray, whilst a high organic content results in a better aerosol formation. This effect can be seen when compounds of varying polarity are analyzed with gradient elution in RP-HPLC. Thus, there are no straight-forward conclusions that can be drawn regarding the differences in ESI ionization efficiency of the porphyrins observed in this study.

\section{Determination of microbial killing efficiency}

The agar plates with nontreated bacteria showed a CFU range between 30 and 300 at dilution factors $10^{-3}$ to $10^{-4}$. The agar plates on which the TE-buffer and ultrasonication treated bacteria were cultivated the CFU count was zero in all dilution steps, yielding a bacterial killing efficiency $\geq 99.98 \%$. The lysis of bacteria is efficient using this method and it is thus assumed that most of the porphyrins are extracted from the bacteria. 
Fig. 2 Chromatogram from the HPLC/MS/MS analysis of a porphyrin standard mixture. UP: uroporphyrin I; 7P: 7carboxylporphyrin I; 6P: 6carboxylporphyrin I; 5P: 5carboxylporphyrin I; CP: coproporphyrin I; MPIX: mesoporphyrin IX; PPIX: protoporphyrin IX

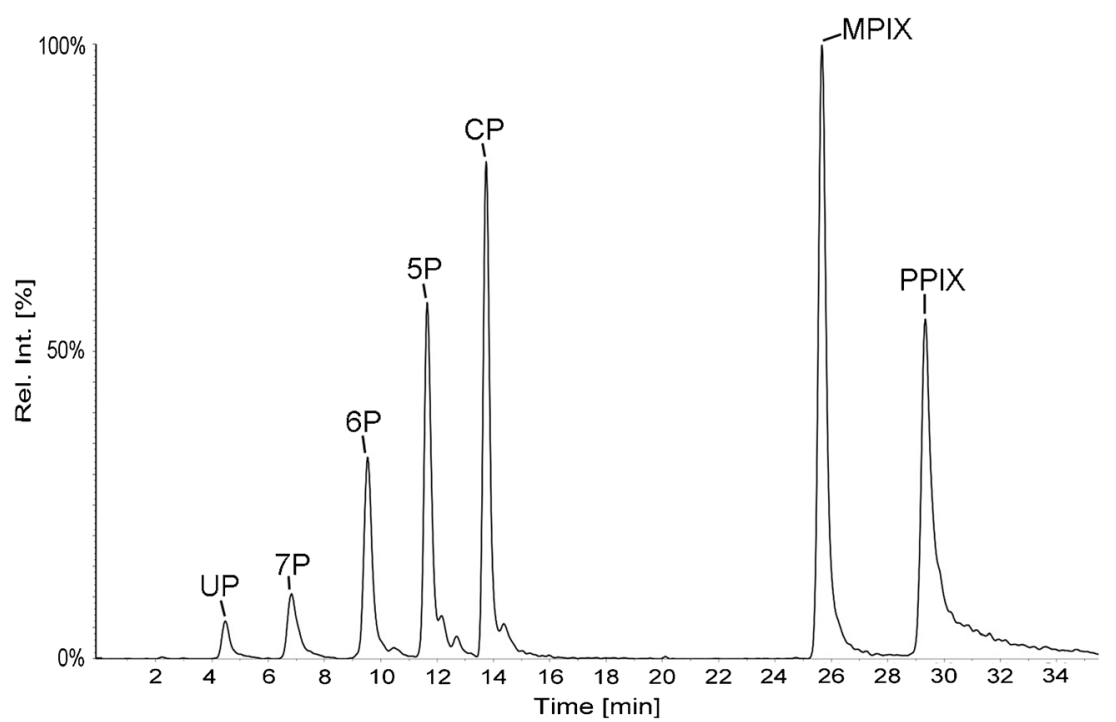

A. actinomycetemcomitans is a gram-negative bacterium, which means that it has an inner and an outer cell membrane, making the bacterium hard to lyse. High lysis efficiency of this bacterium indicates that the method will yield a good porphyrin extraction for other gram-negative, gram-positive and yeast cells as well.

\section{Method validation}

\section{Linearity, $L O D$, and $L O Q$}

The linearity of the calibration curves was high for all the investigated porphyrins demonstrated by coefficients of determination $\left(\mathrm{R}^{2}\right)$ being higher than 0.98 for all the analytes. In Table S1 in the Electronic Supplementary Material (ESM) the linear regression equation and $\mathrm{R}^{2}$ for each porphyrin are listed, and the calibration curve for CPI is shown in Fig. 3.
Coefficients of variation (CV\%) were calculated for retention time and peak area (A) at each concentration level $(n=$ 18). CV\% for area units are mean values from the average at each concentration level in the calibration curve and are listed in ESM Table S2. Retention times showed high stability with $\mathrm{CV} \% \leq 1 \%$ for all analytes. The mean $\mathrm{CV} \%$ in area at six concentration levels was $<10 \%$ for all porphyrins and as low as $3 \%$ for MPIX.

$\mathrm{S} / \mathrm{N}$ ratio, LOD, and LOQ for each porphyrin are summarized in Table 3. LOD ranged between 2.7 and $11.8 \mathrm{fmol}$ (1.5$8.8 \mathrm{pg}$ ) for all porphyrins. Lowest LOD, $2.7 \mathrm{fmol}$ (1.5 pg), was demonstrated by PPIX.

\section{Accuracy, precision, and recovery}

Accuracy, precision, and recovery of the complete analytical method for the investigated porphyrins are sum-

Fig. 3 Calibration curve for CPI

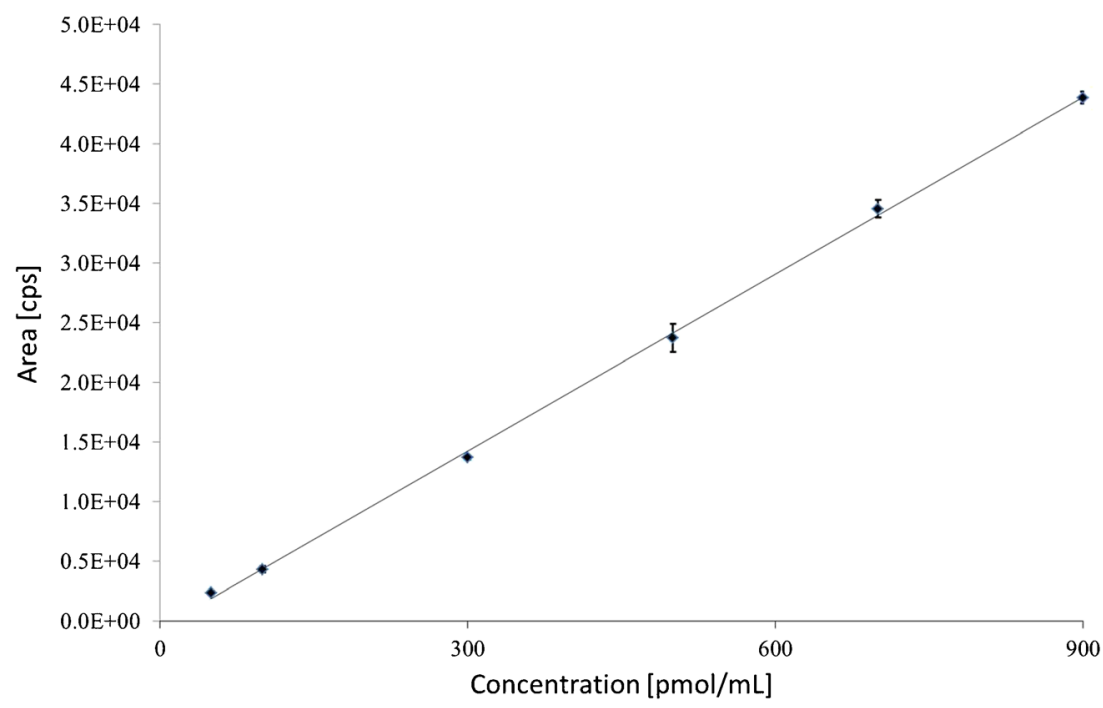


Table 3 S/N, LOD and LOQ calculated using an injected amount of 25 fmol of each porphyrin $(n=3)$

\begin{tabular}{lccc}
\hline Porphyrin & S/N ratio & LOD $[$ fmol injected, $( \pm \mathrm{SD})]$ & LOQ $[$ fmol injected, $( \pm \mathrm{SD})]$ \\
\hline Uroporphyrin I & 9.4 & $7.9( \pm 0.8)$ & $26.5( \pm 2.8)$ \\
7-Carboxylporphyrin I & 6.8 & $11.1( \pm 1.6)$ & $36.9( \pm 5.5)$ \\
6-Carboxylporphyrin I & 6.3 & $11.8( \pm 1.9)$ & $39.4( \pm 6.2)$ \\
5-Carboxylporphyrin I & 19.5 & $3.8( \pm 0.2)$ & $12.8( \pm 0.7)$ \\
Coproporphyrin I & 10.2 & $7.3( \pm 0.7)$ & $24.5( \pm 2.4)$ \\
Mesoporphyrin IX & 11.0 & $6.8( \pm 0.6)$ & $22.8( \pm 2.1)$ \\
Protoporphyrin IX & 27.6 & $2.7( \pm 0.1)$ & $9.1( \pm 0.3)$ \\
\hline
\end{tabular}

marized in Table 4. Intra-day precision was high (RSD $<5 \%$ ) for all porphyrins, including the internal standard compound. Accuracy was also good, demonstrated by a deviation from the reference value of less than $7 \%$ for four of the porphyrins. For the two "extreme" compounds (i.e., those with eight (UPI) and two (PPIX) carboxylic acid substituent groups), the deviation was 13 and $19 \%$, respectively. The recoveries for the porphyrins through the entire sample preparation were high, between 79 and $99 \%$ for four of the porphyrin analytes as well as for the internal standard, and also showing a high reproducibility ( $\mathrm{RSD} \leq 6 \%$ ) for these compounds. Also regarding recoveries, the results for the two extreme compounds, UPI and PPIX, deviated from the other analytes. These two porphyrins showed lower recoveries, 67 and $55 \%$ respectively, with slightly lower reproducibility, 9 and $16 \%$, respectively. Since this group of porphyrins varies widely in polarity, having between two to eight carboxylic acid substituent groups and also a large variation in $\mathrm{pKa}$, it is difficult to obtain a short and efficient method with high recoveries optimal for all the individual porphyrins. The described method does, however, demonstrate a good accuracy with high reproducibility, as well as good recoveries for most compounds with high reproducibility for all the analytes. By investigating the individual parts of the method it was clear that no degradation of porphyrins was caused by the TE digestion or the ultrasonication. The decrease in recovery of the analytes was solely due to the SPE clean-up. This is quite plausible, considering the very large difference in polarity and $\mathrm{pKa}$ values of these analytes yielding a broad elution profile from the SPE.

\section{Matrix effects}

Evaluation of matrix effects is essential for all MS methods since interfering substances in the sample can alter the response leading to ion suppression or ion enhancement. $S$. cerevisiae was used as a model matrix to determine matrix effects of residual cell material in the sample analysis as it is often used as a test organism in chromatographic evaluations [26-29]. Three samples of $S$. cerevisiae (approximately $60 \mathrm{mg}$ per sample) were put through the sample preparation steps, pooled, and spiked with known amounts of UPI, 7PI, 6PI, 5PI, CPI, CPIII, MPIX, and PPIX prior to analysis. Matrix effects were calculated by comparing the response factors for the porphyrins in the spiked extract with those obtained from standard prepared in $6 \mathrm{M}$ formic acid. Endogenously produced amounts of porphyrins in the extracts were subtracted from the spiked samples. The mean matrix effects for the individual porphyrins were UPI $-5 \%$, 7PI $9 \%$, 6PI $-1 \%$, 5PI $2 \%$, CPI $+13 \%$, CPIII $-1 \%$, MPIX $-4 \%$, and for PPIX $-14 \%$. This demonstrates that the matrix effect from residual cell
Table 4 Accuracy, intra-day precision, and recovery $(n=3)$

\begin{tabular}{llll}
\hline Porphyrin & Accuracy $[\%,( \pm \mathrm{SD})]$ & Intra-day precision $[\%]$ & Recovery $[\%,( \pm \mathrm{SD})]$ \\
\hline Uroporphyrin I & $113( \pm 14)$ & 3 & $67( \pm 6)$ \\
7-Carboxylporphyrin I & $93( \pm 1)$ & 1 & $79( \pm 2)$ \\
6-Carboxylporphyrin I & $99( \pm 6)$ & 3 & $88( \pm 5)$ \\
5-Carboxylporphyrin I & $97( \pm 7)$ & 5 & $84( \pm 2)$ \\
Coproporphyrin I & $99( \pm 4)$ & 2 & $99( \pm 3)$ \\
Mesoporphyrin IX & $\mathrm{IS}_{\mathrm{vol}}$ & 4 & $79( \pm 1)$ \\
Protoporphyrin IX & $81( \pm 1)$ & 5 & $55( \pm 9)$
\end{tabular}


Fig. 4 HPLC-MS/MS

chromatogram of porphyrins extracted from

A. actinomycetemcomitans cultivated at $7 \mathrm{~d}$. Parts of chromatogram in dashed boxes are enlarged for better visual effect. Analytes present in the sample: UP (184 ng/g), 7P (28 ng/ g), CPI (6 ng/g), CPIII (20 ng/g), and PPIX (286 ng/g)

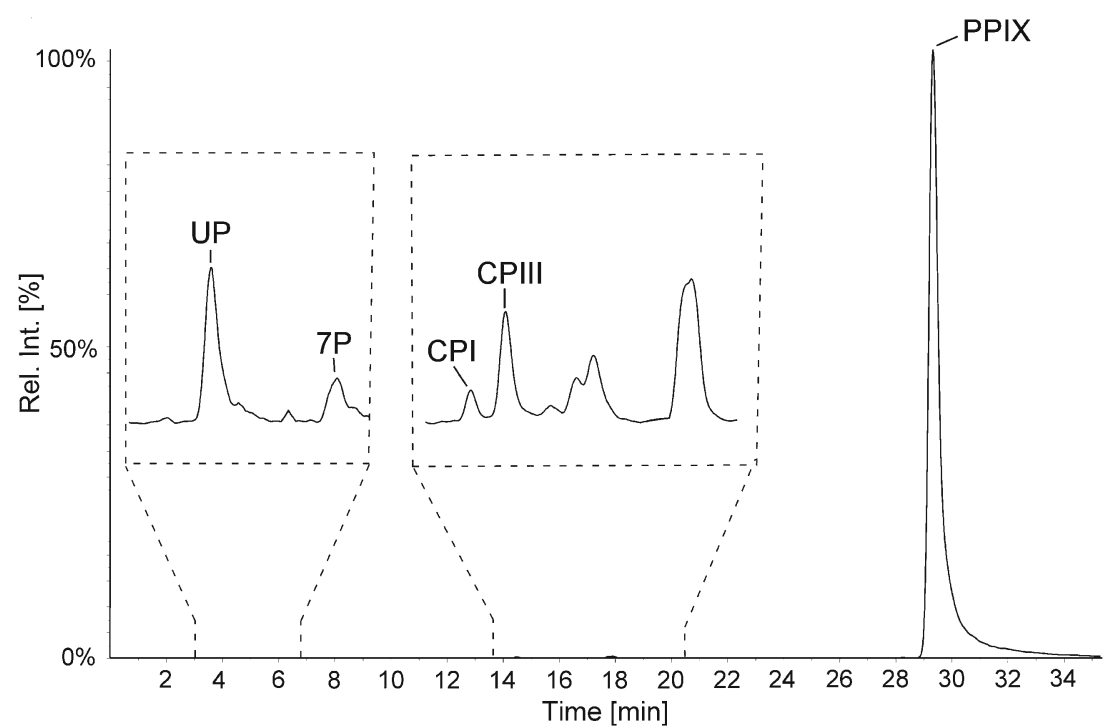

material is low for all the investigated porphyrins using this method.

\section{Porphyrin content in A. actinomycetemcomitans, $P$. gingivalis, and $S$. cerevisiae}

A. actinomycetemcomitans was cultivated on five agar plates and pooled to get an average of endogenously produced porphyrins. Extracts of A. actinomycetemcomitans were analyzed the same day using the described method and porphyrins were identified through retention time and three characteristic SRM transitions in the MS/MS analysis. A typical chromatogram from bacterial sample analysis is shown in Fig. 4, identifying UP, 7P, CPI, CPIII, and PPIX. MPIX is not an intermediate in biosynthesis of heme and could not be detected in any of the analyzed bacteria extracts. This makes it suitable for use as an internal standard. Highest concentrations were PPIX and UP, with concentrations of 286 and $184 \mathrm{ng} / \mathrm{g}$ respectively; 7P, CPI, and CPIII were of lower concentrations with 28,6 , and $20 \mathrm{ng} / \mathrm{g}$, respectively.

P. gingivalis were cultivated anaerobically on five agar plates and pooled. CPI, CPIII, and PPIX were detected and quantified in bacterial extracts, Fig. 5. PPIX had clearly the highest concentration with $214 \mathrm{ng} / \mathrm{g}$, whereas CPI and CPIII had lower concentrations with 4 and $26 \mathrm{ng} / \mathrm{g}$, respectively. Both CP and PPIX have been detected earlier in P. gingivalis [7, 30]. The concentration of porphyrins are in the same range as were found in A. actinomycetemcomitans with the exception of UP and $7 \mathrm{P}$ not being detected. P. gingivalis lacks all of the enzymes involved in the formation of 5-aminolaevulinic
Fig. 5 HPLC-MS/MS

chromatogram of porphyrins extracted from $P$. gingivalis cultivated for $12 \mathrm{~d}$.. Three porphyrins were detected in the sample: CPI (4 ng/g), CPIII (26 ng/g), and PPIX (214 ng/g)

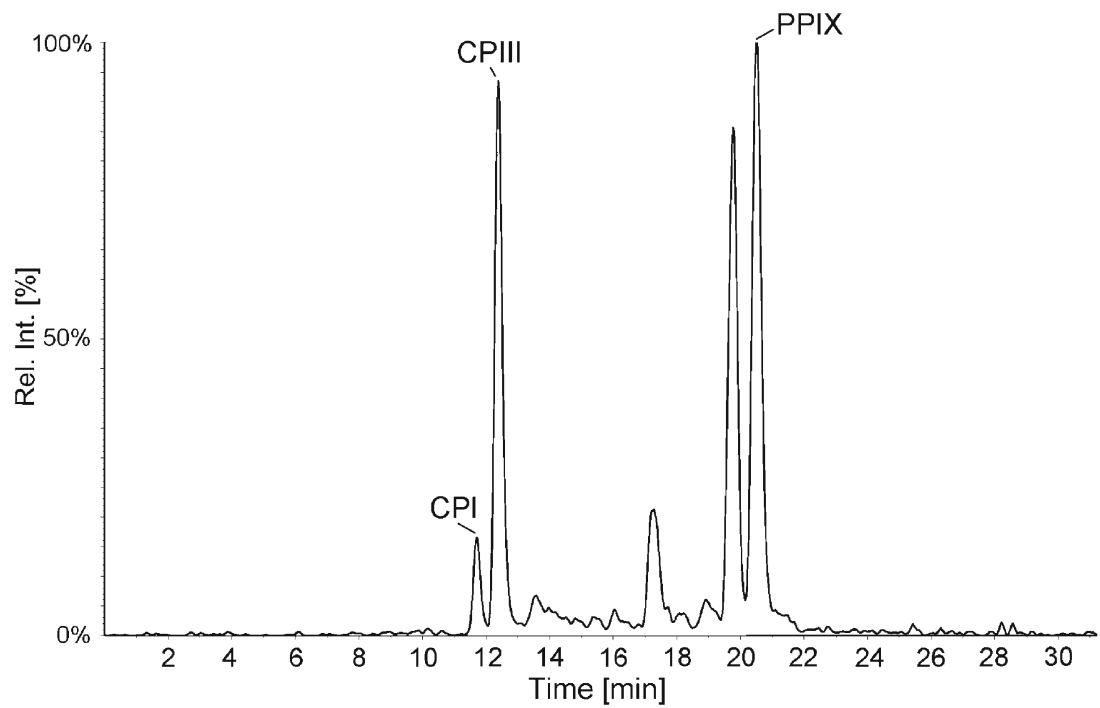


Fig. 6 HPLC-MS/MS

chromatogram of porphyrins extracted from $S$. cerevisiae. Six porphyrins were detected in the sample: UP (19 ng/g), 7P (5 ng/g), 6P (5 ng/g), CPI (11 ng/g), CPIII (79 $\mathrm{ng} / \mathrm{g})$, and PPIX (2 ng/g)

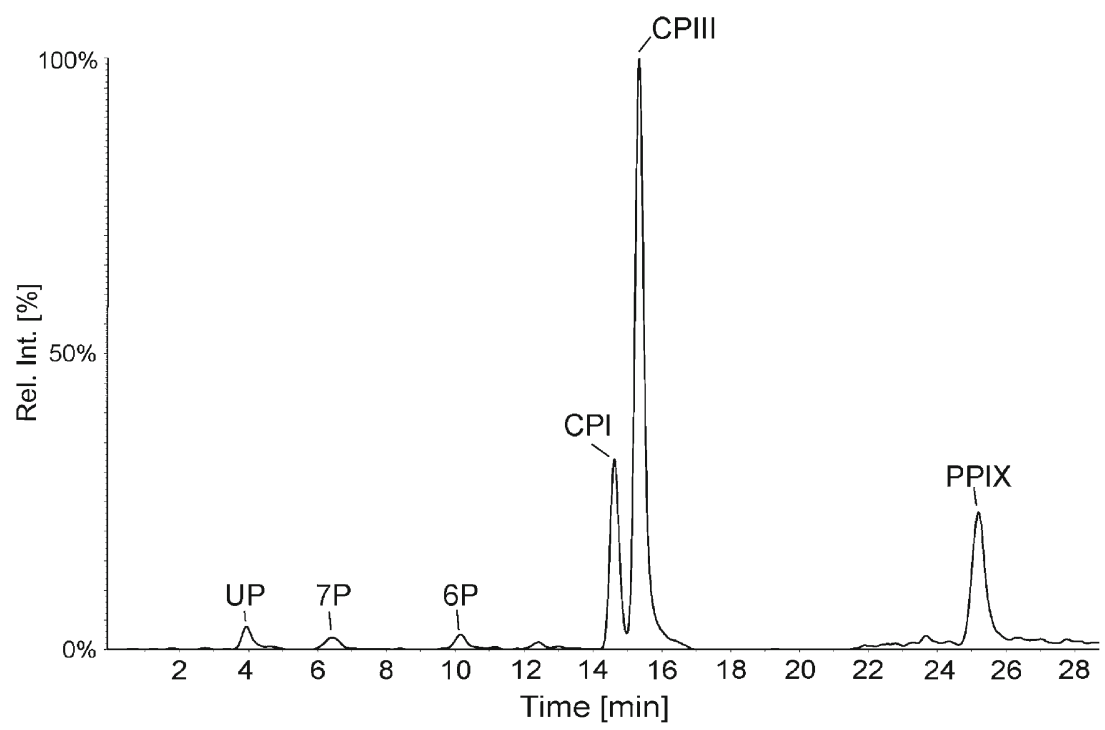

acid (porphyrin precursor) and most of the enzymes involved in the biosynthesis of porphyrins and heme, which are all present in $A$. actinomycetemcomitans and, thus, it is expected that more porphyrins will be found in the latter. $P$. gingivalis does, however, have the enzyme for conversion of CPIII to PPIX as well as ferrochelatase for formation of heme [31]. P. gingivalis is, therefore, dependent on an external source of iron and is able to utilize iron by uptake of organic iron including hemin, hemoglobin, myoglobin, cytochrome $c$, transferrin, as well as inorganic iron in the form of ferric chloride and ferric nitrate [32]. It has been shown that the heme captured on the cell surface are porphyrin-specific, and porphyrins with similar structures to PPIX, including MPIX and deuteroporphyrin IX, bind to the receptors by low affinity interactions [33, 34]. The bacteria could have an uptake of porphyrins from the growth medium, and this can be an explanation as to why CPI and CPIII were detected in $P$. gingivalis despite the fact that they lack enzymes for synthesis of coproporphyrin isomers, but this specific uptake has not been studied. Another explanation could be that $P$. gingivalis has an alternative pathway of heme synthesis similar to that observed in Desulfovibrio vulgaris where the classic route is bypassed but rejoined at CPIII [35, 36].

When $S$. cerevisiae was analyzed, a large number of porphyrins were detected: UP, 7P, 6P, CPI, CPIII, and PPIX. PPIX was the least abundant porphyrin with a concentration of only $2 \mathrm{ng} / \mathrm{g}$, while on the other hand it was the predominant porphyrin in $A$. actinomycetemcomitans and $P$. gingivalis. The most abundant porphyrin in $S$. cerevisiae was CPIII with a concentration of $79 \mathrm{ng} / \mathrm{g}$. A chromatogram from the LC/MS analysis is shown in Fig. 6. Concentrations and percent of porphyrin content for all microbes are summarized in Table 5 .

Table 5 Concentrations of porphyrins in extracts of the oral bacteria A. actinomycetemcomitans and $P$. gingivalis, and baker's yeast $S$. cerevisiae

\begin{tabular}{|c|c|c|c|c|c|c|}
\hline \multirow{2}{*}{$\begin{array}{l}\text { Organism } \\
\text { Porphyrin }\end{array}$} & \multicolumn{2}{|c|}{ A. actinomycetemcomitans } & \multicolumn{2}{|l|}{$P$. gingivalis } & \multicolumn{2}{|l|}{ S. cerevisiae } \\
\hline & Concentration $[\mathrm{ng} / \mathrm{g}]$ & $\%$ of total porphyrin content & Concentration $[\mathrm{ng} / \mathrm{g}]$ & $\%$ of total porphyrin content & Concentration $[\mathrm{ng} / \mathrm{g}]$ & $\%$ of total porphyrin content \\
\hline Uroporphyrin & 184 & 35 & n.d. & - & 19 & 16 \\
\hline 7-Carboxylporphyrin & 28 & 5 & n.d. & - & 5 & 5 \\
\hline 6-Carboxylporphyrin & n.d. & - & n.d. & - & 5 & 4 \\
\hline 5-Carboxylporphyrin & n.d. & - & n.d. & - & n.d & - \\
\hline Coproporphyrin I & 6 & 1 & 4 & 2 & 11 & 9 \\
\hline Coproporphyrin III & 20 & 4 & 26 & 11 & 79 & 65 \\
\hline Mesoporphyrin IX & n.d. & - & n.d. & n.d & n.d & - \\
\hline Protoporphyrin IX & 286 & 55 & 214 & 87 & 2 & 1 \\
\hline Sum: & 524 & 100 & 244 & 100 & 121 & 100 \\
\hline
\end{tabular}

n.d. not detected 


\section{Conclusions}

A method for extraction and analysis of porphyrins from bacteria and yeast has been developed and validated. A. actinomycetemcomitans and $S$. cerevisiae were used for evaluating matrix effects. Highly selective detection using MS/MS of porphyrins in oral bacteria was applied, and the method offers the possibility of accurate characterization of bacterial porphyrin contents. All compounds were well separated, including the isomers CPI and CPIII, using a PFP modified $\mathrm{C} 18$ stationary phase for the HPLC separation. The extraction method is good with efficient cell lysis and high recoveries and reproducibility. Sample clean-up using SPE gave minimal matrix effects for all investigated porphyrins. The method was applied to $P$. gingivalis for determination of porphyrins, showing that the method can be successfully extended for use on other oral bacteria. In P. gingivalis, CPI, CPIII, and PPIX were identified, in consistent with earlier studies. For the first time, to our knowledge, UP, 7P, CPI, CPIII, and PPIX were identified in A. actinomycetemcomitans.

Acknowledgments The authors acknowledge support for this study by the Swedish Research Council, contract no. K2014-70X -22533-01-3.

Open Access This article is distributed under the terms of the Creative Commons Attribution 4.0 International License (http:// creativecommons.org/licenses/by/4.0/), which permits unrestricted use, distribution, and reproduction in any medium, provided you give appropriate credit to the original author(s) and the source, provide a link to the Creative Commons license, and indicate if changes were made.

\section{References}

1. Lim C, Rideout J, Wright D (1983) High-performance liquid chromatography of naturally occurring 8-, 7-, 6-, 5-, and 4-carboxylic porphyrin isomers. J Chromatogr A 282:629-641

2. Bu W, Myers N, McCarty JD, O'Neill T, Hollar S, Stetson PL, Sved DW (2003) Simultaneous determination of six urinary porphyrins using liquid chromatography-tandem mass spectrometry. J Chromatogr B 783:411-423

3. Lim CK (2010) High-performance liquid chromatography and mass spectrometry of porphyrins, chlorophylls and bilins. [Toh Tuck Link] Singapore

4. Li J, Cai Z, Xu S, Liao C, Song X, Chen L (2011) Analysis of urinary porphyrins by high performance liquid chromatographyelectrospray ionization mass spectrometry. J Liquid Chromatogr Related Technol 34:1578-1593

5. Danton M, Lim CK (2006) Porphyrin profiles in blood, urine and faeces by HPLC/electrospray ionization tandem mass spectrometry. Biomed Chromatogr 20:612-621

6. Hindmarsh JT, Oliveras L, Greenway DC (1999) Plasma porphyrins in the porphyrias. Clin Chem 45:1070-1076

7. Soukos NS, Som S, Abernethy AD, Ruggiero K, Dunham J, Lee C, Doukas AG, Goodson JM (2005) Phototargeting oral blackpigmented bacteria. Antimicrob Agents Chemother 49:1391-1396

8. Božek P, Hutta M, Hrivnáková B (2005) Rapid analysis of porphyrins at low $\mathrm{ng} / \mathrm{l}$ and $\mu \mathrm{g} / \mathrm{l}$ levels in human urine by a gradient liquid chromatography method using octadecylsilica monolithic columns. J Chromatogr A 1084:24-32

9. Mateo R, Castells G, Green AJ, Godoy C, Cristòfol C (2004) Determination of porphyrins and biliverdin in bile and excreta of birds by a single liquid chromatography-ultraviolet detection analysis. J Chromatogr B 810:305-311

10. Macours P, Cotton F (2006) Improvement in HPLC separation of porphyrin isomers and application to biochemical diagnosis of porphyrias. Clin Chem Lab Med 44:1433-1440

11. Melanson JE, Lucy CA (2002) Enhanced detection of porphyrins by capillary electrophoresis-laser induced fluorescence. Electrophoresis 23:1689-1694

12. Ashkenazi H, Malik Z, Harth Y, Nitzan Y (2003) Eradication of Propionibacterium acnes by its endogenic porphyrins after illumination with high intensity blue light. FEMS Immunol Med Microbiol 35:17-24

13. Romiti R, Schaller M, Jacob K, Plewig G (2000) High-performance liquid chromatography analysis of porphyrins in Propionibacterium acnes. Arch Dermatol Res 292:320-322

14. Alammari M, Smith P, de Josselin de Jong E, Higham S (2013) Quantitative light-induced fluorescence (QLF): a tool for early occlusal dental caries detection and supporting decision making in vivo. J Dent 41:127-132

15. Borisova E, Uzunov T, Avramov L (2006) Laser-induced autofluorescence study of caries model in vitro. Laser Med Sci 21:34-41

16. van der Veen MH, Thomas RZ, Huysmans MC, de Soet JJ (2006) Red autofluorescence of dental plaque bacteria. Caries Res 40:542-545

17. Coulthwaite L, Pretty IA, Smith PW, Higham SM, Verran J (2006) The microbiological origin of fluorescence observed in plaque on dentures during QLF analysis. Caries Res 40:112-116

18. Bjurshammar N, Johannsen A, Buhlin K, Tranæus S, Östman C (2012) On the red fluorescence emission of Aggregatibacter actinomycetemcomitans. Open Stomatology 4:299-306

19. Kjeldstad B, Johnsson A, Sandberg S (1984) Influence of pH on porphyrin production in Propionibacterium acnes. Arch Dermatol Res 276:396-400

20. Lee WL, Shalita AR, Poh-Fitzpatrick MB (1978) Comparative studies of porphyrin production in Propionibacterium acnes and Propionibacterium granulosum. J Bacteriol 133:811-815

21. Feuerstein O, Persman N, Weiss EI (2004) Phototoxic effect of visible light on Porphyromonas gingivalis and Fusobacterium nucleatum: an in vitro study. Photochem Photobiol 80:412-415

22. Konig K, Teschke M, Sigusch B, Glockmann E, Eick S, Pfister W (2000) Red light kills bacteria via photodynamic action. Cell Mol Biol [Noisy-le-grand] 46:1297-1303

23. Fukui M, Yoshioka M, Satomura K, Nakanishi H, Nagayama M (2008) Specific-wavelength visible light irradiation inhibits bacterial growth of Porphyromonas gingivalis. J Periodont Res 43:174-178

24. Brown SB, Shillcock M, Jones P (1976) Equilibrium and kinetic studies of the aggregation of porphyrins in aqueous solution. Biochem J 153:279-285

25. Fenn JB (1993) Ion formation from charged droplets: roles of geometry, energy, and time. J Am Soc Mass Spectrom 4:524-535

26. Maynard DM, Masuda J, Yang X, Kowalak JA, Markey SP (2004) Characterizing complex peptide mixtures using a multi-dimensional liquid chromatography-mass spectrometry system: Saccharomyces cerevisiae as a model system. J Chromatogr B 810:69-76

27. Saleh A, Bruno O, Granelli I, Edlund P (2014) Digestion of enolase and carbonic anhydrase as model proteins for therapeutic proteins in blood plasma with immobilized thermolysin and quantification of some of the peptides by LC/LC-MS/MS. Chromatographia 77:59-74

28. Knittelfelder OL, Weberhofer BP, Eichmann TO, Kohlwein SD, Rechberger GN (2014) A versatile ultra-high performance LC-MS method for lipid profiling. J Chromatogr B 951:119-128

29. Sun T, Wetzel SJ, Johnson ME, Surlow BA, Patton-Vogt J (2012) Development and validation of a hydrophilic interaction liquid 
chromatography-tandem mass spectrometry method for the quantification of lipid-related extracellular metabolites in Saccharomyces cerevisiae. J Chromatogr B 897:1-9

30. Henry CA, Judy M, Dyer B, Wagner M, Matthews JL (1995) Sensitivity of Porphyromonas and Prevotella species in liquid media to argon laser. Photochem Photobiol 61:410-413

31. Roper JM, Raux E, Brindley AA, Schubert HL, Gharbia SE, Shah HN, Warren MJ (2000) The enigma of cobalamin (vitamin B12) biosynthesis in Porphyromonas gingivalis. Identification and characterization of a functional corrin pathway. J Biol Chem 275:40316-40323

32. Bramanti TE, Holt SC (1991) Roles of porphyrins and host iron transport proteins in regulation of growth of Porphyromonas gingivalis W50. J Bacteriol 173:7330-7339

33. Paramaesvaran M, Nguyen KA, Caldon E, McDonald JA, Najdi S, Gonzaga G, Langley DB, DeCarlo A, Crossley MJ, Hunter N, Collyer CA (2003) Porphyrin-mediated cell surface heme capture from hemoglobin by Porphyromonas gingivalis. J Bacteriol 185:2528-2537

34. Hunter N, Nguyen K, McDonald JA, Quinn MJ, Langley DB, Crossley MJ, Collyer CA (2002) Structural requirements for recognition of essential porphyrin by Porphyromonas gingivalis. JPP 6:774-782

35. Ishida T, Yu L, Akutsu H, Ozawa K, Kawanishi S, Seto A, Inubushi T, Sano S (1998) A primitive pathway of porphyrin biosynthesis and enzymology in Desulfovibrio vulgaris. Proc Natl Acad Sci U S A 95: 4853-4858

36. Bali S, Palmer DJ, Schroeder S, Ferguson SJ, Warren MJ (2014) Recent advances in the biosynthesis of modified tetrapyrroles: the discovery of an alternative pathway for the formation of heme and heme d 1. Cell Mol Life Sci 71:2837-2863

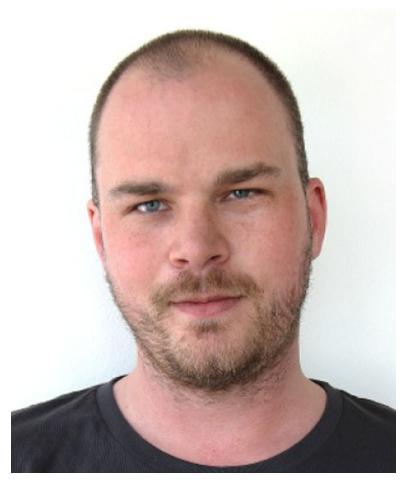

Jonas Fyrestam PhD student at the Department of Environmental Science and Analytical Chemistry, Stockholm University, Sweden. His research focuses mainly on method development and determination of porphyrins in microorganisms, utilizing analytical methods such as HPLC-MS/MS.

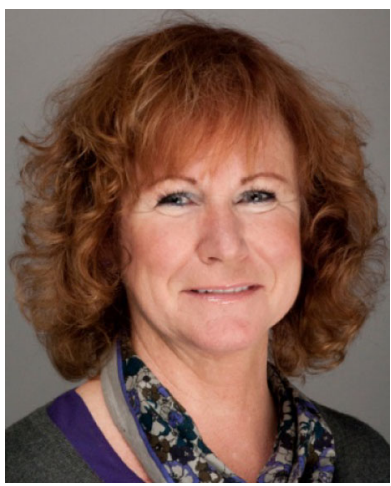

Nadja Bjurshammar PhD student and Director of studies at the Dental Hygiene Programme, Department of Dental Medicine, Division of Periodontology, Karolinska Institutet, Sweden. Bjurshammar has extensive practical experience in prevention and clinical treatment of periodontal diseases. She is working with invitro phototherapy treatment of bacteria, bacteria cultivation, and QLF fluorescence analysis.

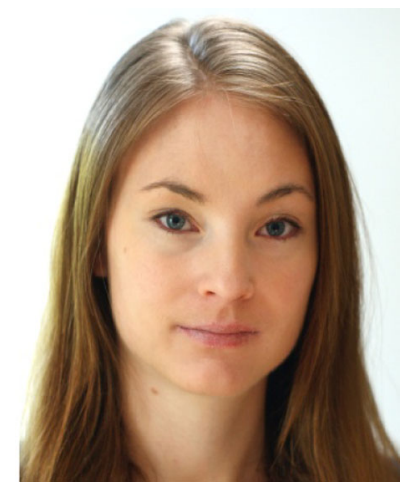

Elin Paulsson is at present working as a chemist at the Department of Aquatic Sciences and Assessment, Swedish University of Agricultural Sciences, Uppsala, Sweden. Paulsson has a master degree in analytical chemistry from Stockholm University. She is currently working with analysis of pesticides using HPLC/MS/MS and GC/MS.

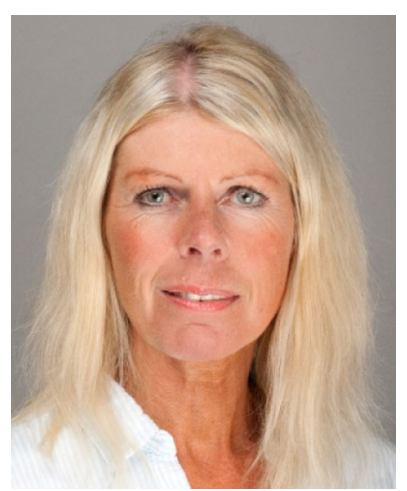

Annsofi Johannsen Associate Professor, Head of the unit for Dental Hygienist Programme at the Department of Dental Medicine, Division of Periodontology, Karolinska Institutet, Sweden She has extensive experience in research in the fields of inflammation, risk factors for periodontal diseases, as well as for Crohns disease.

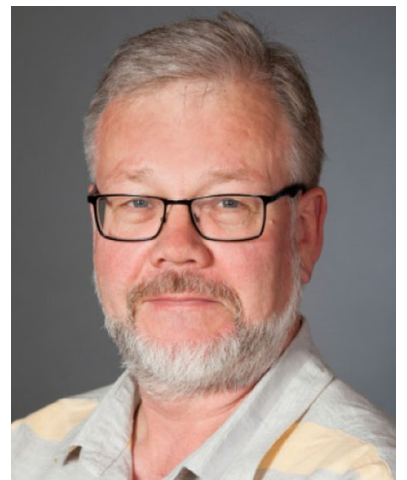

Conny Östman Professor, Analytical and Toxicological Chemistry, Department of Environmental Science and Analytical Chemistry, Stockholm University, Sweden. Östman has 35 years of experience in analytical chemistry research working with method development and coupled analytical techniques with focus on chromatography and mass spectrometry. $\mathrm{He}$ has been working with compounds such as $\mathrm{PAH}, \mathrm{PCB}$, PBDE, organophosphates, phthalates etc. in indoor and outdoor environments as well as for personal exposure measurement. Recent research areas are chemicals in textiles, breath analysis, and phototherapy and porphyrins in oral bacteria. 